\title{
Odd Integers $N$ With Five Distinct Prime Factors for Which $2-10^{-12}<\sigma(N) / N<2+10^{-12}$
}

\section{By Masao Kishore*}

\begin{abstract}
We make a table of odd integers $N$ with five distinct prime factors for which $2-10^{-12}<\sigma(N) / N<2+10^{-12}$, and show that for such $N|\sigma(N) / N-2|>10^{-14}$. Using this inequality, we prove that there are no odd perfect numbers, no quasiperfect numbers and no odd almost perfect numbers with five distinct prime factors. We also make a table of odd primitive abundant numbers $N$ with five distinct prime factors for which $2<\sigma(N) / N<2+2 / 10^{10}$.
\end{abstract}

1. A positive integer $N$ is called perfect, quasiperfect (QP), or almost perfect according as $\sigma(N)=2 N, 2 N+1$, or $2 N-1$, respectively, where $\sigma(N)$ is the sum of the positive divisors of $N$. While twenty-four even perfect numbers are known, no odd perfect (OP) numbers, no QP numbers, and no almost perfect numbers except a power of 2 are known.

In this paper we make a table of odd integers $N$ with five distinct prime factors for which

$$
2-10^{-12}<\sigma(N) / N<2+10^{-12},
$$

and we show that for such $N$

$$
|\sigma(N) / N-2|>10^{-14} \text {. }
$$

Using this inequality, we prove that there are no $\mathrm{OP}, \mathrm{QP}$, or odd almost perfect (OAP) numbers with five distinct prime factors.

$N$ is called primitive abundant if $N$ is abundant $(\sigma(N)>2 N)$ and every proper divisor $M$ of $N$ is deficient $(\sigma(M)<2 M)$. In 1913 Dickson [4] published a table of odd primitive abundant numbers with less than five distinct prime factors. In this paper we also make a table of odd primitive abundant numbers $N$ with five distinct prime factors for which

$$
2<\sigma(N) / N<2+2 / 10^{10} .
$$

2. Throughout this paper we let $N=\Pi_{i=1}^{r} p_{i}^{a_{i}}$ where $3 \leqslant p_{1}<\cdots<p_{r}$ are primes and $a_{i}$ 's are positive integers. $p_{i}^{a_{i}}$ is called a component of $N$.

We define

$$
\begin{aligned}
a(p) & =\min \left\{a \mid p^{a+1}>10^{12}\right\}, \\
\omega(N) & =r, \\
S(N) & =\sigma(N) / N=\prod_{i=1}^{r}\left(p_{i}^{a_{i}+1}-1\right) / p_{i}^{a_{i}}\left(p_{i}-1\right),
\end{aligned}
$$

Received December 6, 1976; revised March 23, 1977.

AMS (MOS) subject classifications (1970). Primary $10 \mathrm{~A} 20$.

* This paper is a part of the author's doctoral dissertation which was submitted to Princeton University in August 1977 and directed by Professor J. Chidambaraswamy of the University of Toledo. copyright $\odot$ 1978. American Mathematical Society 


$$
\begin{aligned}
& A(N)=\left[\prod_{a_{i}<a\left(p_{i}\right)} S\left(p_{i}^{\left.a_{i}\right)}\right]\left[\prod_{a_{i} \geqslant a\left(p_{i}\right)} S\left(p_{i}^{a\left(p_{i}\right)}\right)\right],\right. \\
& B(N)=\left[\prod_{a_{i}<a\left(p_{i}\right)} S\left(p_{i}^{a_{i}}\right)\right]\left[\prod_{a_{i} \geqslant a\left(p_{i}\right)} p_{i} /\left(p_{i}-1\right)\right], \\
& L\left(p^{a}\right)= \begin{cases}{\left[10^{12} \log S\left(p^{a}\right)\right] / 10^{12}} & \text { if } a<a(p), \\
{\left[10^{12} \log p /(p-1)\right] / 10^{12}} & \text { if } a \geqslant a(p),\end{cases}
\end{aligned}
$$

where [ ] is the greatest integer function. We note that if $p, q$ are primes with $p>$ $q$ and $a, b$ are positive integers then

$$
S\left(p^{a}\right)=\left(p^{a+1}-1\right) / p^{a}(p-1)<p /(p-1)=\lim _{a \rightarrow \infty} S\left(p^{a}\right) \leqslant(q+1) / q \leqslant S\left(q^{b}\right),
$$

and so $L\left(p^{a}\right) \leqslant L\left(q^{b}\right)$ and $A(N) \leqslant S(N) \leqslant B(N)$. Hence, we have

LEMMA 1. (a) If $A(N)>2-10^{-12}$ and $B(N)<2+10^{-12}, N$ satisfies (1).

(b) If $A(N) \leqslant 2-10^{-12}<B(N)<2+10^{-12}$, some $N$ satisfies (1).

(c) If $2-10^{-12}<A(N)<2+10^{-12} \leqslant B(N)$, some $N$ satisfies (1).

(d) If $A(N)<2-10^{-12}$ and $2+10^{-12}<B(N)$, some $N$ may satisfy (1).

(e) If $2+10^{-12}<A(N)$ or $B(N)<2-10^{-12}, N$ does not satisfy (1).

In Lemmas 2 through 5 we assume that $N$ satisfies $(1)$ and $\omega(N)=5$.

LEMMA 2.

$$
0.6931471805544<\sum_{i=1}^{5} L\left(p_{i}^{b_{i}}\right)<0.6931471805655
$$

where $b_{i}=\min \left\{a_{i}, a\left(p_{i}\right)\right\}$.

Proof. Suppose $p^{a}$ is a component of $N$. If $a<a(p)$, then

$$
\left|\log S\left(p^{a}\right)-L\left(p^{a}\right)\right|<10^{-12} .
$$

If $a \geqslant a(p)$, then $p^{a+1}>10^{12}$ and

$$
\begin{aligned}
10^{-12} & >\log p /(p-1)-L\left(p^{a}\right)>\log S\left(p^{a}\right)-L\left(p^{a}\right) \geqslant \log S\left(p^{a}\right)-\log p /(p-1) \\
& =\log \left(1-1 / p^{a+1}\right)=-\sum_{i=1}^{\infty} 1 / i\left(p^{a+1}\right)^{i}>-1 /\left(p^{a+1}-1\right) \geqslant-10^{-12} .
\end{aligned}
$$

Hence

$$
\left|\log S\left(p^{a}\right)-L\left(p^{a}\right)\right|<10^{-12}
$$

Since (1) holds,

$$
\begin{aligned}
0.6931471805544 & <\log \left(2-10^{-12}\right)-5 / 10^{12} \\
& <\sum_{i=1}^{5} \log S\left(p_{i}^{a_{i}}\right)-5 / 10^{12}<\sum_{i=1}^{5} L\left(p_{i}^{b_{i}}\right) \\
& <\sum_{i=1}^{5} \log S\left(p_{i}^{a_{i}}\right)+5 / 10^{12}<\log \left(2+10^{-12}\right)+5 / 10^{12}
\end{aligned}
$$

$$
<0.6931471805655 \text {. Q.E.D. }
$$


LemMA 3. $p_{1}=3, p_{2} \leqslant 11$ and $p_{3} \leqslant 41$.

Proof. Lemma 3 follows from the following inequalities:

$$
\begin{aligned}
& \frac{5}{4} \frac{7}{6} \frac{11}{10} \frac{13}{12} \frac{17}{16}<2-10^{-12}, \\
& \frac{3}{2} \frac{13}{12} \frac{17}{16} \frac{19}{18} \frac{23}{22}<2-10^{-12}, \\
& \frac{3}{2} \frac{5}{4} \frac{43}{42} \frac{47}{46} \frac{53}{52}<2-10^{-12} \text {. Q.E.D. }
\end{aligned}
$$

LEMMA 4. $p_{4}<5000$.

Proof. Suppose $N$ satisfies (1) and $p_{4} \geqslant 5003$. Then

$$
\begin{aligned}
0 & \leqslant L\left(p_{5}^{b_{5}}\right) \leqslant L\left(p_{4}^{b_{4}}\right)<\log S\left(p_{4}^{b_{4}}\right)+10^{-12} \\
& <\log p_{4} /\left(p_{4}-1\right)+10^{-12}<1 /\left(p_{4}-1\right)+10^{-12} \\
& <0.0002 .
\end{aligned}
$$

Hence by (3)

$$
0.69274<\sum_{i=1}^{3} L\left(p_{i}^{b_{i}}\right)<0.69315 .
$$

A computer (PDP11 at the University of Toledo) was used to find $\Pi_{i=1}^{3} p_{i}^{b_{i}}$ satisfying (4), but there were none. Q.E.D.

Similarly, we can prove 36551083.

LeMmA 5. $p_{5}<3000000$, or $\Pi_{i=1}^{4} p_{i}^{b_{i}}=3^{7} 5^{6} 17^{2} 233$ and $36549767 \leqslant p_{5} \leqslant$

The computer was used to find $N=\Pi_{i=1}^{5} p_{i}^{a_{i}}$ satisfying $a_{i} \leqslant a\left(p_{i}\right)$, Lemmas 3,4 , 5, and Lemma 2 or Lemma 1(b), (c), (d), with the result given in Table 1.

Lemma 6. Suppose $N=\Pi_{i=1}^{5} p_{i}^{a_{i}}$ and $M=\Pi_{i=1}^{5} p_{i}^{b_{i}}$ where $b_{i}=\min \left\{a_{i}, a\left(p_{i}\right)\right\}$.

If $M=3^{23} 5^{12} 17^{6} 257^{4} 65521,|S(N)-2|>5 / 10^{13}$;

if $M=3^{8} 5^{14} 17^{3} 251 \cdot 1884529,|S(N)-2|>2 / 10^{14}$;

if $M=3^{8} 5^{9} 17^{3} 251 \cdot 1579769,|S(N)-2|>3 / 10^{13}$;

if $M=3^{8} 5^{8} 17^{9} 269^{4} 4153^{3},|S(N)-2|>4 / 10^{14}$;

if $\Pi_{i=1}^{4} p_{i}^{b_{i}}=3^{7} 5^{6} 17^{2} 233,|S(N)-2|>10^{-14}$.

In all other cases $|S(N)-2|>10^{-13}$.

Proof. The first part of Lemma 6 follows from the following inequalities:

$$
\begin{aligned}
& S\left(3^{23} 5^{12} 17^{6} 257^{4} 65521\right)<2-5 / 10^{13}, \\
& S\left(3^{23} 5^{12} 17^{6} 257^{5} 65521\right)>2+1 / 10^{12}, \\
& S\left(3^{8} 5^{14} 17^{3} 251\right) 1884529 / 1884528<2-2 / 10^{14}, \\
& S\left(3^{8} 5^{9} 17^{3} 251 \cdot 1579769\right)<2-4 / 10^{13}, \\
& S\left(3^{8} 5^{9} 17^{3} 251 \cdot 1579769^{2}\right)>2+3 / 10^{13}, \\
& S\left(3^{8} 5^{8} 269^{4}\right) 17 / 16 \cdot 4153 / 4152<2-4 / 10^{14}, \\
& S\left(3^{8} 5^{8} 17^{9} 269^{5} 4153^{3}\right)>2+3 / 10^{13}, \\
& S\left(3^{7} 5^{6} 17^{2} 233 \cdot 36550379\right)>2+5 / 10^{14},
\end{aligned}
$$


and

$$
S\left(3^{7} 5^{6} 17^{2} 233\right) 36550429 / 36550428<2-10^{-14} \text {. }
$$

Suppose $|S(N)-2| \leqslant 10^{-13}$. Then (1) holds, and so $N$ is given in Table 1 ; however, for every $N$ in Table 1 except for those given above $S(N) \leqslant B(N)<2-10^{-13}$, or $S(N) \geqslant A(N)>2+10^{-13}$. Q.E.D.

We have proved

THEOREM. If $N$ is an odd integer with $\omega(N)=5,|\sigma(N) / N-2|>10^{-14}$.

3. We used a similar method to find odd primitive abundant numbers $N=$ $\Pi_{i=1}^{5} p_{i}^{a_{i}}$ for which (2) holds, with the result given in Table 2 in the microfiche. Table 2 includes odd primitive abundant numbers $N$ with $\omega(N)=5$ one of whose component $p^{a}$ is greater than $10^{10}$; for, letting $M=N / p^{a}$, we have

$$
\begin{aligned}
2 & <\sigma(N) / N=\sigma(M) \sigma\left(p^{a}\right) / M p^{a}=\sigma(M)\left(p \sigma\left(p^{a-1}\right)+1\right) / M p^{a} \\
& =\sigma\left(M p^{a-1}\right) / M p^{a-1}+\sigma(M) / M p^{a}<2+2 / 10^{10},
\end{aligned}
$$

showing that (2) holds.

4. Suppose $N$ is an odd integer such that $\sigma(N)=2 N+A$. If $|A| N \mid \leqslant 10^{-14}$, then by our Theorem $\omega(N) \geqslant 6$. We give three examples of such $N$.

Suppose $N$ is OP. Sylvester (1888), Dickson (1913), and Kanold (1949) proved that $\omega(N) \geqslant 5$. From our Theorem we have

Proposition 1. If $N$ is $O P, \omega(N) \geqslant 6$.

This fact was also proved by Gradšteīn (1925), Kühnel (1949) and Webber (1951). Pomerance [1] (1972) and Robbins (1972) proved that $\omega(N) \geqslant 7$, and Hagis [2] proved that $\omega(N) \geqslant 8$.

Proposition 2. If $N$ is $Q P, \omega(N) \geqslant 6$.

Proof. By [3] if $N$ is QP, then $N$ is an odd perfect square, $\omega(N) \geqslant 5$ and $N>$ $10^{20}$. Hence $2<S(N)=2+1 / N<2+10^{-20}$, and so by Theorem $\omega(N) \geqslant 6$. Q.E.D.

LEMMA 7. If $N$ is $O A P, p N$ is primitive abundant for some $p \mid N$.

Proof. Suppose $N=\Pi_{i=1}^{r} p_{i}^{a_{i}}$ is OAP, and choose $j$ so that $\sigma\left(p_{j}^{a_{j}}\right) \geqslant \sigma\left(p_{i}^{a_{i}}\right)$ for every $i$. Letting $p=p_{j}, a=a_{j}$ and $L=N / p^{a}$, we have

$$
\begin{aligned}
2 p^{a} L-1 & =\sigma(N)=\sigma\left(p^{a}\right) \sigma(L) \\
& =\left(1+p \sigma\left(p^{a-1}\right)\right) \sigma(L)=\sigma(L)+p \sigma\left(p^{a-1}\right) \sigma(L) .
\end{aligned}
$$

Hence $p \mid \sigma(L)+1$. If $p=\sigma(L)+1$, then

$$
\begin{aligned}
\sum_{i=1}^{a+1} p^{i} & =\sigma\left(p^{a}\right) p=\sigma\left(p^{a}\right) \sigma(L)+\sigma\left(p^{a}\right) \\
& =\sigma(N)+\sigma\left(p^{a}\right)=2 p^{a} L-1+\sigma\left(p^{a}\right)=2 p^{a} L+\sum_{i=1}^{a} p^{i},
\end{aligned}
$$

or $p^{a+1}=2 p^{a} L$, showing that $N=2^{a}$. Since $N$ is OAP, $p \neq \sigma(L)+1$, and so $p<$ $\sigma(L)$ because $p \mid \sigma(L)+1$. Then

$$
\begin{aligned}
\sigma(p N) & =\sigma\left(p^{a+1}\right) \sigma(L)=\left(1+p \sigma\left(p^{a}\right)\right) \sigma(L) \\
& =\sigma(L)+p \sigma(N)=\sigma(L)+2 p N-p>2 p N,
\end{aligned}
$$

showing that $p N$ is abundant. 
Suppose $M$ is a proper divisor of $p N$. If $p^{a+1} \nmid M$, then $M$ is a divisor of $N$, and $M$ is deficient because

$$
S(M) \leqslant S(N)=2-1 / N<2 .
$$

Suppose $p^{a+1} \mid M$. Then for some $k, p_{k}^{a_{k}} \nmid M$. Letting $q=p_{k}$ and $b=a_{k}$, we have $\sigma\left(p^{a}\right) \geqslant \sigma\left(q^{b}\right)$, or

$$
\sum_{i=1}^{b} q^{i} \leqslant \sum_{i=1}^{a} p^{i}<\sum_{i=1}^{a+1} p^{i}
$$

Hence

$$
\left(1 / p^{a+1}\right) \sum_{i=0}^{b-1} q^{-i}<\left(1 / q^{b}\right) \sum_{i=0}^{a} p^{-i},
$$

and by adding $\Sigma_{i=0}^{a} p^{-i} \Sigma_{i=0}^{b-1} q^{-i}$ to both sides we obtain

$$
\sum_{i=0}^{a+1} p^{-i} \sum_{i=0}^{b-1} q^{-i}<\sum_{i=0}^{a} p^{-i} \sum_{i=0}^{b} q^{-i}
$$

or $S\left(p^{a+1}\right) S\left(q^{b-1}\right)<S\left(p^{a}\right) S\left(q^{b}\right)$. Then

$$
\begin{aligned}
S(M) & \leqslant S\left(p^{a+1}\right) S\left(q^{b-1}\right) \prod_{i \neq j, k} S\left(p_{i}^{a_{i}}\right) \\
& <S\left(p^{a}\right) S\left(q^{b}\right) \prod_{i \neq j, k} S\left(p_{i}^{a_{i}}\right)=S(N)<2,
\end{aligned}
$$

showing that $M$ is deficient. Q.E.D.

LEMMA 8. If $N=\Pi_{i=1}^{r} p_{i}^{a_{i}}$ is $O A P, a_{i}$ is even. If $p_{1}=3, a_{1} \geqslant 12$.

Proof. Suppose $N$ is OAP, $p^{a}$ is a component of $N, q$ is a prime and $q \mid \sigma\left(p^{a}\right)$. Since $\sigma(N)=2 N-1$ is odd and $\sigma\left(p^{a}\right) \mid \sigma(N), \sigma\left(p^{a}\right)=\Sigma_{j=0}^{a} p^{j}$ is odd. Hence $a$ is even. Since $q \mid 2 \sigma(N)=4 N-2$ and $4 N$ is a perfect square, $(2 \mid q)=1$, where $(2 \mid q)$ is the Legendre symbol, and so $q \equiv 1$ or $7(\bmod 8)$ because $(2 \mid q)=(-1)^{\left(q^{2}-1\right) / 8}$. Also $\sigma\left(p^{a}\right) \equiv 1$ or $7(\bmod 8)$, for, otherwise, $\sigma\left(p^{a}\right)$ would have a prime factor $\equiv 3$ or 5 $(\bmod 8)$.

Suppose $p=3$ and $a=2 e$. Then $\sigma\left(3^{2 e}\right) \equiv 1+4 e \equiv 1$ or $7(\bmod 8)$, or $e \equiv$ $0(\bmod 2)$. Hence $a=4,8,12, \ldots$; however, $a \neq 4$ or 8 because $11 \mid \sigma\left(3^{4}\right), 11 \equiv$ $3(\bmod 8), 13 \mid \sigma\left(3^{8}\right)$ and $13 \equiv 5(\bmod 8)$. Q.E.D.

Proposition 4. If $N$ is $O A P, \omega(N) \geqslant 6$.

Proof. Suppose $N=\Pi_{i=1}^{r}$ is OAP. Then by Lemma $7 p N$ is primitive abundant for some $p \mid N$. If $3 \nmid N, \omega(N) \geqslant 7$, for, otherwise,

$$
2<S(p N)<\prod_{i=1}^{r} \frac{p_{i}}{p_{i}-1} \leqslant \frac{5}{4} \frac{7}{6} \frac{11}{10} \frac{13}{12} \frac{17}{16} \frac{19}{18}<2 .
$$

Suppose $3 \mid N$. Then $3^{12} \mid p N$ by Lemma 8 . According to the table of odd primitive abundant numbers $M$ with fewer than five distinct prime factors in [4] $3^{12} \nmid M$. 
Hence $\omega(N) \geqslant 5$, and $N \geqslant 3^{12} 5^{2} 7^{2} 11^{2} 13^{2}>10^{13}$. Then $2>S(N)=2-1 / N>$ $2-10^{-13}$, and by Lemma $6 \omega(N) \geqslant 6$. Q.E.D.

For other results on QP and OAP see [3], [5], [6], [7] and [8].

Computer time for Tables 1 and 2 was over four hours.

\section{TABLE 1}

$$
N=\Pi_{i=1}^{5} p_{i}^{a_{i}} \text { for which } 2-10^{-12}<\sigma(N) / N<2+10^{-12(a)}
$$

\begin{tabular}{|c|c|c|c|c|}
\hline$p_{1}^{b_{1}}$ & $p_{2}^{b_{2}}$ & $p_{3}^{b} 3$ & $p_{4}^{b_{4}}$ & $p_{5}^{b_{5}}$ \\
\hline $3^{25}$ & $5^{5}$ & $17^{7}$ & 251 & $570407^{(b)}$ \\
\hline $3^{23}$ & $5^{12}$ & $17^{6}$ & $257^{4}$ & $65521^{(c)}$ \\
\hline $3^{22}$ & $5^{5}$ & $17^{6}$ & 251 & $569659^{2}$ \\
\hline \multirow[t]{2}{*}{$3^{21}$} & $5^{9}$ & $17^{9}$ & $257^{4}$ & $65099^{2(b)}$ \\
\hline & $5^{5}$ & $17^{5}$ & 251 & 557273 \\
\hline $3^{20}$ & $5^{14}$ & $17^{5}$ & $257^{4}$ & $65357^{(b)}$ \\
\hline $3^{19}$ & $5^{3}$ & $17^{3}$ & 181 & $57149^{2}$ \\
\hline \multirow[t]{2}{*}{$3^{18}$} & $5^{5}$ & $17^{5}$ & 251 & $557017^{2}$ \\
\hline & & $17^{4}$ & 251 & $406811^{2}$ \\
\hline $3^{16}$ & $5^{5}$ & $17^{8}$ & 251 & $567943^{2}$ \\
\hline $3^{12}$ & $5^{5}$ & $17^{5}$ & 251 & $412943^{2}$ \\
\hline $3^{11}$ & $5^{12}$ & $17^{9}$ & $257^{3}$ & $58337^{(c)}$ \\
\hline $3^{10}$ & $5^{10}$ & $17^{9}$ & $257^{3}$ & $47791^{2(c)}$ \\
\hline $3^{9}$ & $7^{3}$ & $13^{5}$ & $19^{2}$ & $1009643^{(b)}$ \\
\hline \multirow[t]{10}{*}{$3^{8}$} & $5^{16}$ & $17^{8}$ & $257^{4}$ & $15137^{2(c)}$ \\
\hline & $5^{14}$ & $17^{3}$ & 251 & $1884527^{(c)}$ \\
\hline & & & & 1884529 \\
\hline & $5^{13}$ & $17^{3}$ & 25.1 & $1884061^{(c)}$ \\
\hline & $5^{11}$ & $17^{3}$ & 251 & 1870207 \\
\hline & $5^{9}$ & $17^{3}$ & 251 & 1579769 \\
\hline & $5^{8}$ & $17^{9}$ & $269^{4}$ & $4153^{3(d)}$ \\
\hline & $5^{3}$ & $19^{9}$ & $83^{6}$ & 493277 \\
\hline & & $19^{8}$ & $83^{3}$ & $488203^{2}$ \\
\hline & & $19^{7}$ & $83^{4}$ & 493201 \\
\hline $3^{7}$ & $5^{6}$ & $17^{2}$ & 233 & (e) \\
\hline
\end{tabular}

Note: (a) If $b_{i}=a\left(p_{i}\right)$ and $c>0, N p_{i}^{c}$ also satisfies (1). See Lemma 1 (a).

(b) See Lemma 1(b). (c) See Lemma 1(c). (d) See Lemma 1(d).

(e) $36549767<p_{5}<36551083$. 
Department of Mathematics University of Toledo

Toledo, Ohio 43606

1. C. POMERANCE, "Odd perfect numbers are divisible by at least seven distinct primes," Acta Arith., v. 25, 1974, pp. 265-299.

2. P. HAGIS, JR., "Every odd perfect number has at least eight prime factors," Abstract \#720-10-14, Notices Amer. Math. Soc., v. 22, 1975, p. A-60.

3. H. L. ABBOT, C. E. AULL, E. BROWN \& D. SURYANARAYANA, "Quasiperfect numbers," Acta Arith., v. 22, 1973, pp. 439-447.

H. L. ABBOT, C. E. AULL, E. BROWN \& D. SURYANARAYANA, Corrections to the paper “Quasiperfect numbers," Acta Arith., v. 29, 1976, pp. 427-428.

4. L. E. DICKSON, "Finiteness of the odd perfect and primitive abundant numbers with $n$ distinct prime factors," Amer. J. Math., v. 35, 1913 , pp. 413-422.

5. M. KISHORE, "Quasiperfect numbers are divisible by at least six distinct prime factors," Abstract \#75T-A113, Notices Amer. Math. Soc., v. 22, 1975 , p. A-441.

6. M. KISHORE, “Odd almost perfect numbers," Abstract \#75T-A92, Notices Amer. Math. Soc., v. 22, 1975 , p. A-380.

7. R. P. JERRARD \& N. TEMPERLEY, “Almost perfect numbers," Math. Mag., v. 46, 1973, pp. 84-87.

8. J. T. CROSS, “A note on almost perfect numbers," Math. Mag., v. 47, 1974, pp. $230-$ 231. 